\title{
2,5-二噻吩基吡咯衍生物的合成与应用研究进展
}

\author{
邓君 ${ }^{a}$ 傅相锴*,a,b 王 刚 ${ }^{a}$ 黄 静 ${ }^{a}$ 吴 柳 ${ }^{a}$ 邹晓川 ${ }^{c}$ \\ ( ${ }^{a}$ 西南大学化学化工学院应用化学研究所 重庆市应用化学市级重点实验室 重庆 400715) \\ $\left({ }^{b}\right.$ 三峡库区生态环境教育部重点实验室 重庆 400715) \\ ( ${ }^{c}$ 重庆教育学院生物与化学工程系 重庆 400067)
}

\begin{abstract}
摘要 2,5-二噻吩基吡咯衍生物具有良好的环境稳定性和电化学性质, 在光电子领域有广泛的应用前景. 综述了 $2,5-$ 二 噻吩基吡咯衍生物中噻吩环和吡咯环上不同位置取代衍生物的合成方法, 并对 2,5-二噻吩基吡咯衍生物在电致变色器 件、光电子器件、传感器等方面的应用进行了概述.
\end{abstract}

关键词 2,5-二噻吩基吡咯衍生物; 合成; 应用; 光电功能材料; 电化学性能

\section{Progress in Synthesis and Application of 2,5-Dithienylpyrrole Derivatives}

\author{
Deng, Jun ${ }^{a}$ \\ Fu, Xiangkai*,a,b \\ Wang, Gang ${ }^{a}$ \\ Huang, Jing ${ }^{a}$ \\ $\mathrm{Wu}, \mathrm{Liu}^{a}$ \\ Zou, Xiaochuan ${ }^{c}$ \\ ( ${ }^{a}$ College of Chemistry and Chemical Engineering, Research Institute of Applied Chemistry, Southwest China University, \\ Key Laboratory of Applied Chemistry of Chongqing Municipality, Chongqing 400715) \\ ( ${ }^{b}$ Key Laboratory of Eco-environments in Three Gorges Region, Ministry of Education, Chongqing 400715) \\ ( ${ }^{c}$ Department of Biological \& Chemical Engineering, Chongqing Education College, Chongqing 400067)
}

\begin{abstract}
Dithienylpyrrole derivatives could be widely used in the optoelectronics field due to their good environmental stability and electrical chemical properties. In this review, the synthetic methods of 2,5-dithienylpyrrole derivatives substituted on different position of thiophene group and pyrrole group are summarized and their applications in electrochromic devices, optoelectronic devices and sensors are presented as well.
\end{abstract}

Keywords 2,5-dithienylpyrrole derivatives; synthesis; application; optoelectronic functional material; electrochemical property

自从 1977 年科学家发现用 $\mathrm{AsF}_{5}$ 或 $\mathrm{I}_{2}$ 掺杂的聚乙炔 具有优异的导电性 ${ }^{[1 \sim 4]}$ 至今, 导电聚合物的发展已有 30 多年的历史, 对它们的开发和研究一直是国内外高分子 化学工作者的研究热点, 这类聚合物现已逐步成为具有 潜在应用前景的一种新型功能材料. 聚吡咯类、聚噻吩 类、聚苯胺类和聚苯基乙炔类等有机导电高分子是目前 研究比较活跃的几类电活性材料, 其中聚吡咯和聚噻吩 因具有高电导率、良好的氧化还原可逆性、随电压快速 变换颜色以及良好的环境稳定性等特点而备受关注, 在
太阳能电池 ${ }^{[5]}$ 、神经探针 ${ }^{[6]}$ 、电致变色材料和器件 ${ }^{[7]}$ 的开 发等方面具有良好的应用前景.

大量的文献报道聚噻吩和聚吡咯衍生物是通过化 学氧化法或者电化学氧化法获得的, 然而, 在氧化噻 吩、吡咯单体的同时, 又会导致聚合物自身的过氧化 ${ }^{[8]}$; 虽然可以通过降低氧化电位或严格控制氧化剂用量来 杜绝这个现象，但又会导致它们的加工和光学等性能变 得更差, 进而限制了它们的应用. 研究发现, 含有杂环 的二聚体或三聚体(比如二噻吩、三噻吩、二噻吩基吡

\footnotetext{
*E-mail: fxk@swu.edu.cn

Received November 10, 2011; revised December 19, 2011; published online January 6, 2012.

Project supported by the National Ministry of Science and Technology Innovation Fund for High-tech Small and Medium Enterprise Technology (No. 09C26215112399) and the National Ministry of Human Resources and Social Security Start-up Support Projects for Students Returned to Business [Office of Human Resources and Social Security Issued 2009(143)].

国家科技部科技中小型企业技术创新基金(No. 09C26215112399)及国家人力资源和社会保障部留学人员回国创业启动支持计划 [No. 人社厅发 2009 (143 号)]资助项目.
} 
咯等)可以在不损害其加工性能和光学性能的情况下降 低其氧化还原电位, 获得较低的能隙 $\left(E_{\mathrm{g}}\right)$, 且聚合物仍 能保持良好的电致变色性能和成膜性能. 正因为如此, 越来越多研究者开始关注二噻吩基吡咯衍生物的合成 研究与应用. 1987 年, Ferraris 和 Skiles ${ }^{[9]}$ 首次提出用 2,5二噻吩基吡咯衍生物(SNS)单体直接聚合得到比例确定 的 2,5-二噻吩基吡咯共聚物(PSNS). 至今, 众多科研工 作者通过不同的方法合成了一系列 2,5-二噻吩基吡咯衍 生物 ${ }^{[10 ~ 15]}$, 并研究了其物理化学性能. 随着近年来有机 合成技术的飞速发展, 固相法和各种新型催化剂广泛应 用到 2,5-二噻吩基吡咯衍生物的合成, 新的合成方法不 断出现, 反应条件更为温和, 合成成本也愈来愈低. 近 年来, Toppare 和 Cihaner 等对这类化合物做了较多的研 究, 结果发现这些化合物都具有优异的电致变色性能, 如色彩丰富、稳定性高、循环寿命长、易于加工成膜等 优点, 预示其在电致变色器件、传感器、发光二极管、 太阳能电池等方面有广泛的应用前景. 然而, 迄今为止, 对于 2,5-二噻吩基吡咯衍生物在合成与应用两个方面的 研究成果鲜有评述, 基于此, 本文就近年来 2,5-二噻吩 基吡咯衍生物的合成以及在电致变色器件、光电子器 件、传感器等方面的研究进展作一综述.

\section{1 常规合成方法}

\subsection{Knorr-Paal 缩合反应}

\subsection{1 直接 Knorr-Paal 缩合反应}

1,4-二(2-噻吩基)-1,4-丁二酮与伯胺通过 Knorr-Paal 缩合反应 ${ }^{[16]}$ 生成 2,5 -二噻吩基吡咯衍生物. 一般认为, 这个反应在酸催化条件下是通过 “加成-消除” 的过程 实现的 ${ }^{[17,18]}$. 首先是 1,4 -二羰基化合物 1 与胺 $\left(\mathrm{RNH}_{2}\right)$ 发 生亲核加成，并随即失去一分子水生成烯胺中间体 ${ }^{[17]}$, 然后烯胺发生分子内亲核加成, 再失去一分子水得到 2,5-二噻吩基吡咯衍生物 2. Scheme 1 所示路线是合成

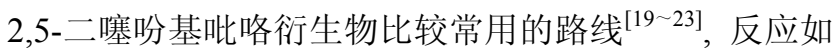
下:

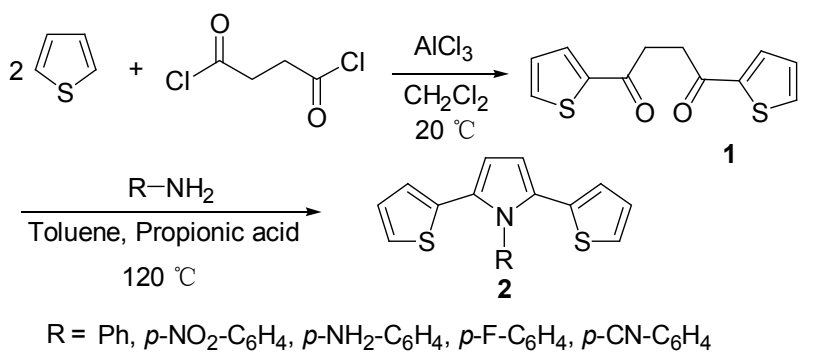

Scheme 1

\subsection{2 偶合反应 + Knorr-Paal 缩合反应}

1993 年, Niziurski-Mann 等 ${ }^{[24]}$ 报道 1-(2-噻吩基)-1-
丙酮在 $\mathrm{Cu}(\mathrm{Tf})_{2}$ 的催化下，与它的烯醇化合物进行偶合， 从而制备出 2,5-二噻吩基吡咯衍生物 3 (Scheme 2).

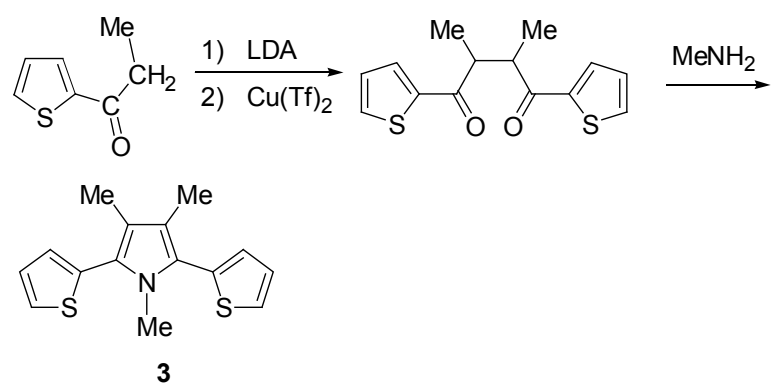

\section{Scheme 2}

\subsubsection{Stetter 反应 + Knorr-Paal 缩合反应}

类似地, Sorensen, Ogura, Niziurski-Mann 等 ${ }^{[25 ~ 27]}$ 利 用曼尼希碱 4 与醛类化合物 $\mathbf{5}$ 或 $\mathbf{6}$, 发生 Stetter 反应，生 成 1,4-二羰基酮化合物 7 或 8 , 再分别与伯胺发生缩合 生成目标产物 9 或 10. 循环伏安法表明, 这类物质的 $\pi$ 共轭体系具有良好的共面性, 失去 $\pi$ 电子的能力也很强 (Scheme 3).
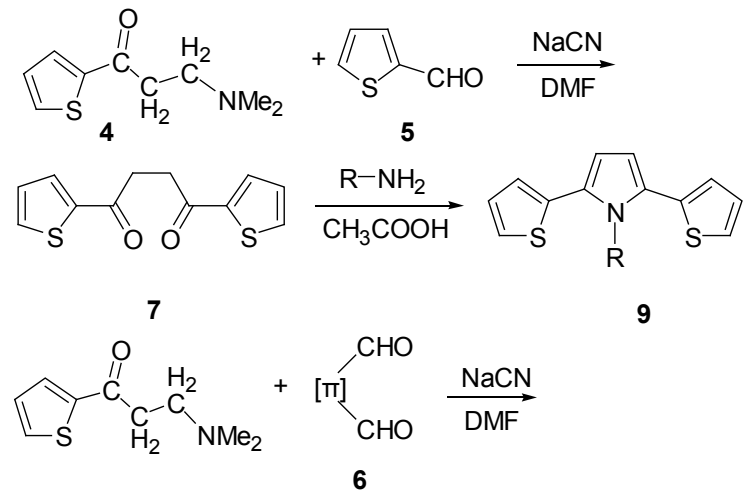<smiles>O=C(CCC(=O)c1cccs1)[In]C(=O)CCC(=O)c1cccs1</smiles><smiles>[R]NC(C)[C@H](O)OC(C)(C)N</smiles>

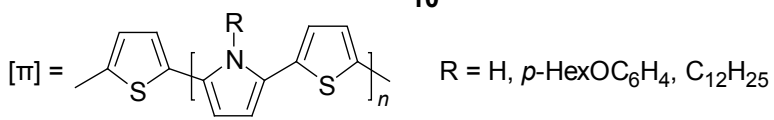

Scheme 3

2009 年, Fujii 等 ${ }^{[28]}$ 为了避免使用 Stetter 反应中的剧 毒物质氰化钠，利用 $\alpha$-溴甲基化合物 11 和 2,5 -二乙酰基 噻吩 12 在 $\mathrm{ZnCl}_{2}-t-\mathrm{BuOH}-\mathrm{Et}_{2} \mathrm{NH}$ 中发生交叉偶联，生成 四酩 13. 然后用甲基胺的醋酸溶液与四酮 13 作用缩合 后生成目标化合物 14. 这些化合物具有较高的空穴迁 移率，可在场效应管中可以作为很好的 $\mathrm{p}$ 型半导体，并 
且具有类似混合五聚体的线性结构(Scheme 4).
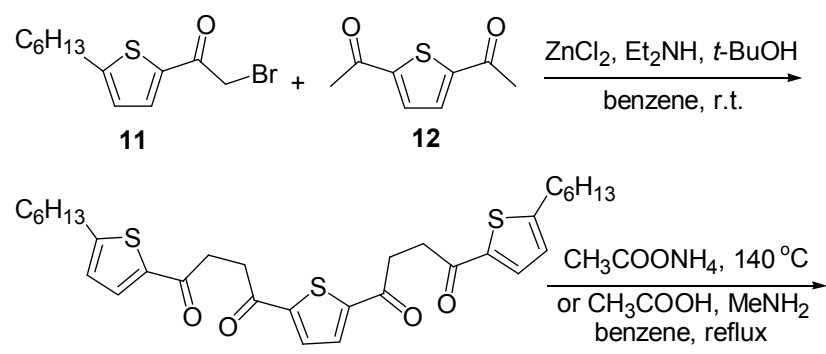

13

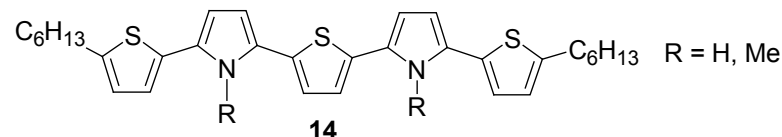

Scheme 4

\section{2 环加成反应}

\subsection{1 亚胺与炔的 $1,3-$ 偶极环加成}

1,3-偶极环加成反应是一类重要的有机反应, 通常 用它来实现五元杂环化合物的合成 ${ }^{[29]}$. St-Cyr 等 ${ }^{[30,31]}$ 用 亚胺 15, 酰氯化物 16 、炔 17 以及邻苯二酚基苯基膦“一 锅煮” 反应关环生成目标产物 $\mathbf{1 8}$ (Eq. 1). 邻苯二酚基苯 基膦为 1,3-偶极环加成试剂, 它可以与炔发生环加成反 应形成吡咯, 而且有助于提高目标产物的产率.

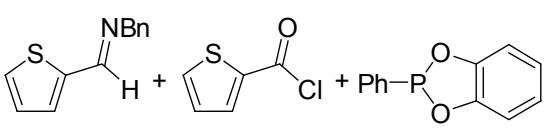

15

16

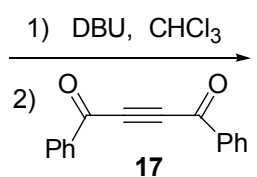

$$
\text { (18) }
$$

传统的合成方法使用有机锂作为亲核试剂会发生 副反应, 且反应需要在极低的温度下才能进行. 2008 年, $\mathrm{Naka}$ 等 ${ }^{[32]}$ 采用炔丙基甲硅烷基醚 19、亚胺 $\mathbf{2 0}$ 以及季膦 盐在无金属催化的反应条件下, 应用一锅法也可得到目 标产物 21, 产率为 $80 \%$ (Eq. 2).

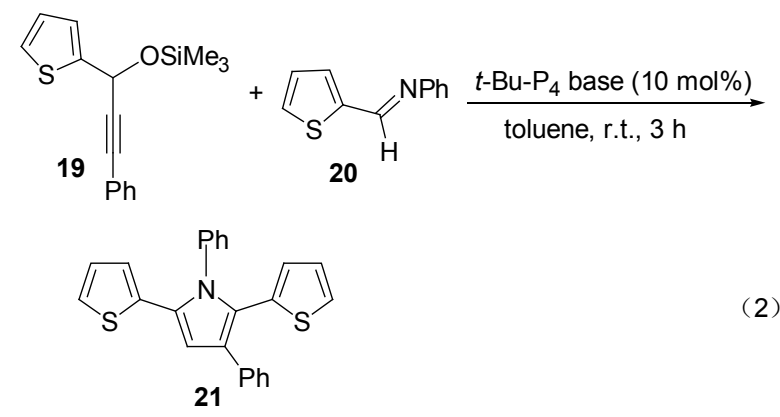

\subsection{2 二炔与伯胺的环加成反应}

2010 年, Zheng 等 ${ }^{[33]}$ 采用苯胺与二炔 22 在氯化亚铜 的催化下, 形成一个烯炔中间体, 最终以 $93 \%$ 的产率合 成了目标产物 23 (Eq. 3).

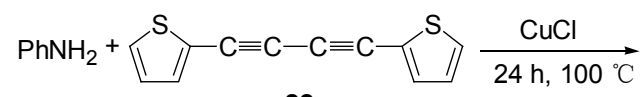

22

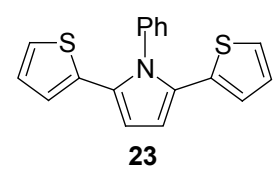

\subsubsection{1,3-二酮、醛和胺的环合反应}

2008 年, Dou 等 ${ }^{\left[{ }^{34]}\right.}$ 报道不对称的 1,3-二酮 24、醛 25 和胺 26 在钛(IV)氯和钐粉作诱导剂的条件下, 环合生成 目标产物 27. 与传统合成方法相比, 这种新方法具有反 应时间短 $(15 \mathrm{~min})$ 、产量高、操作方便、高选择性等优 点(Eq. 4).

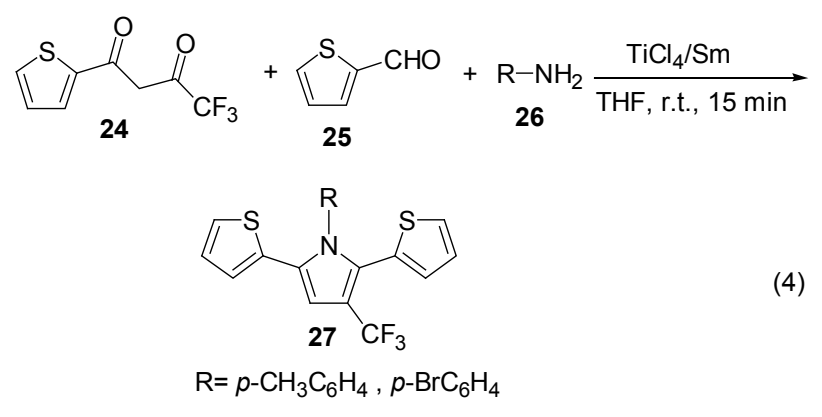

\section{3 在钯金属催化下的偶联反应}

偶联反应是形成 $\mathrm{C}-\mathrm{C}$ 键的基本反应, 在钯催化下 几乎各种亲电试剂都能和有机烷在十分温和的条件下 发生偶联, 反应产率高, 且区域选择性和立体选择性强, 特别是该反应能够允许许多活性官能团存在, 是合成复 杂有机分子的理想反应，已成功地应用于许多天然产物 和关键中间体的合成中 ${ }^{[35]}$.

2003 年, Ono 等 ${ }^{[36]}$ 通过羧酸化合物 $\mathbf{2 8}$ 与 $\mathrm{KI}$ 和 $\mathrm{I}_{2}$ 混 合后，在现制的 $\mathrm{NaHCO}_{3}$ 溶液中反应，即可得到二碘吡 咯化合物 29, 然后再以 $\mathrm{Pd}\left(\mathrm{PPh}_{3}\right)_{4}$ 催化 Suzuki 偶联反应, 将噻吩基引入到吡咯环中, 得到了目标产物 $\mathbf{3 0}$, 产率达 $70 \%$ (Scheme 5). Suzuki 偶联反应的优势就是具有强的 底物适应性及官能团容忍性, 以及形成了噻吩基硼酸及 酯过渡的中间体，让反应更容易进行. 2008 年，Dang 等 ${ }^{\left[{ }^{[7]}\right.}$ 也利用此方法合成了 2,5-二噻吩基吡咯衍生物 31 .

2006 年, Byrne 等 ${ }^{[38]}$ 采用吡咯的溴代衍生物与丁锡 烷基噻吩在钯催化下发生 Stille 交叉偶联反应，可制备 2,5-二噻吩基吡咯衍生物 32. 这一反应在室温下就能 进行, 对底物的兼容性较好、反应选择性也较高. 2009 


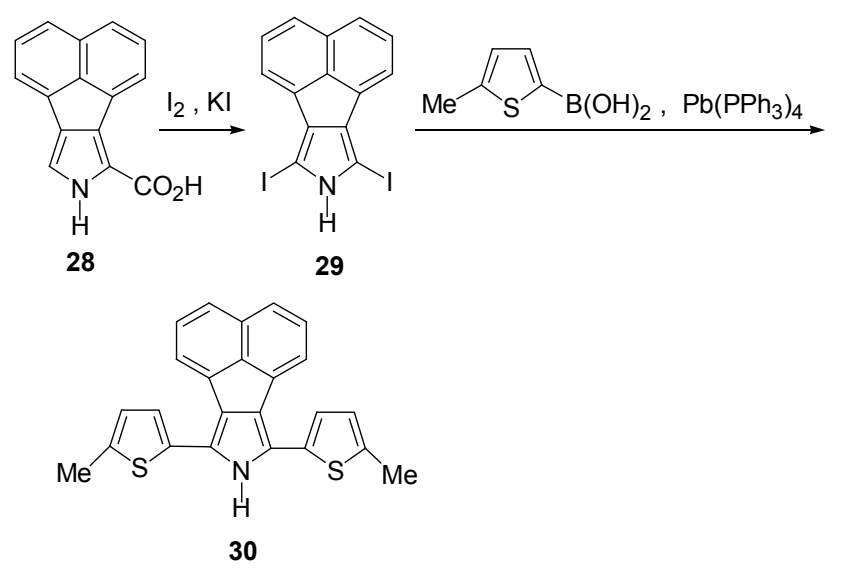

绾<smiles>Cn1c(-c2cccs2)c(Br)c(Br)c1-c1cccs1</smiles>

31

\section{Scheme 5}

年, Kang 等 ${ }^{[39]}$ 也采用此方法, 在 $\operatorname{Pd}\left(\mathrm{PPh}_{3}\right)_{4}$ 催化下, 成功 合成了 2,5-二噻吩基吡咯衍生物 33 (Scheme 6).
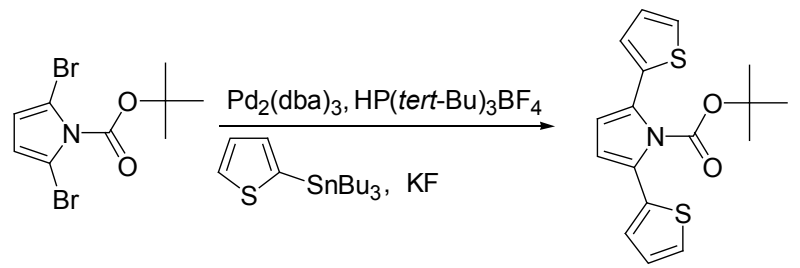

32
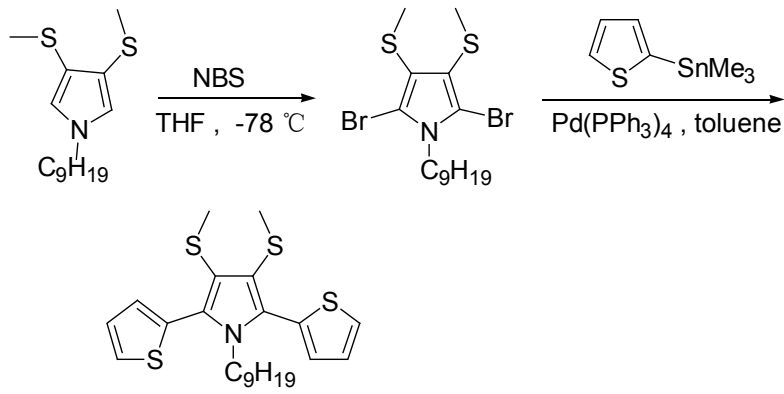

33

\section{Scheme 6}

\section{4 杂环亚胺-烯胺的氧化反应}

1996年, Lehuede 等 ${ }^{[40]}$ 将杂环亚胺一烯胺化合物溶于 乙腈溶液中, 然后用四乙酸铅进行氧化, 合成了目标产 物 34 (Eq. 5).<smiles>[R]C=C(N)c1ccc(P(=O)(O)OC(C)=O)s1</smiles>

\section{2 其它合成方法}

\section{1 固相合成}

固相合成是一种以树脂作为载体的合成技术，最初 用于肽及核酸的研究 ${ }^{[41]}$, 发展至今已三十余年. 由于反 应时键连在固相载体上的底物结构处于适当的溶剂中, 与外加构件分子及试剂分子在固液两相交界处进行有 关的化学反应，反应后只需滤除未链接于固相载体上的 杂质，最后经适当反应切断 linker 键，便将目标产物目 标化合物从固相载体上释放出来 ${ }^{[42]}$. 近年来, 在有机小 分子合成中，作为一门前沿技术引起化学家的广泛关 注，以独特的优势显示出广阔的应用前景 ${ }^{[43,44]}$.

2002 年, Kobayashi 等 ${ }^{[45]}$ 用(1-羟基烯丙基)噻吩基羧 酸 35, $N, N^{\prime}$-二异丙基碳二酰亚胺(DIC), $N$-甲基吡咯烷酮 $(\mathrm{NMP})$, 在 $\mathrm{CH}_{2} \mathrm{Cl}_{2}-\mathrm{DMF}$ 溶液中, 与 Wang 聚苯乙烯树脂 连接起来，用 Dess-Martin 试剂，在常温下，反应 $4 \mathrm{~h}$ ，将 羟基化合物 36 氧化生成酮 37. 烯酤 37 与芳醛发生 Stetter 反应生成 1,4-二羰基酮化合物 38. 然后在 $\mathrm{CH}_{2} \mathrm{Cl}_{2}$ 在溶剂中，在三氟乙酸(TFA)的作用下，脱去树脂. 最后 在甲醇溶剂中，与胺的作用发生 Knorr-Paal 缩合反应， 得到 4 种目标产物 39 (Scheme 7).
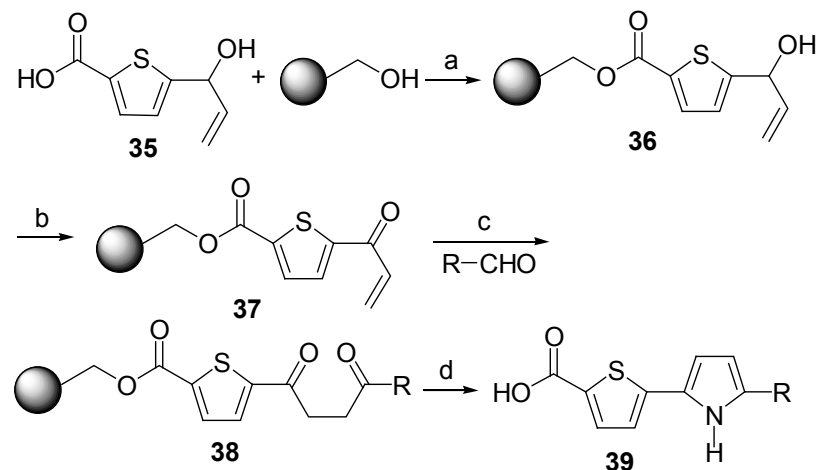

$\mathrm{R}=7$ - $n$-propylbenzo[b]thiophene-2-yl,

7-chloro-4-methylbenzo[b]thiophene-2-yl,

7-chlorobenzo[b]thiophene-2-yl,

4,7-dichlorobenzo[b]thiophene-2-yl

Reagents and conditions: (a) DIC, NMP, $\mathrm{CH}_{2} \mathrm{Cl}_{2}-\mathrm{DMF}(\mathrm{V}: \mathrm{V}=3: 1$ ), r.t., 24 h; (b) Dess-Martin reagent, DMF, r.t., 4 h; (c) $\mathrm{Et}_{3} \mathrm{~N}$, DMF, 65 ${ }^{\circ} \mathrm{C}, 3 \mathrm{~h}$; (d) TFA, $\mathrm{CH}_{2} \mathrm{Cl}_{2}$; (e) $\mathrm{NH}_{4} \mathrm{OAc}, \mathrm{MeOH}, 70{ }^{\circ} \mathrm{C}, 15 \mathrm{~h}$

\section{Scheme 7}

\section{2 微波合成}

微波最早被人们认识并应用在军事通讯领域，而在 有机合成中的研究始于 1986 年, Gedye 等 ${ }^{[46,47]}$ 发现在微 
波辐射下 4-氯基苯氧离子与氯苠的 $\mathrm{S}_{\mathrm{N}} 2$ 亲核取代反应速 率提高 1240 倍, 且产率也有明显的提高. Danks 等 ${ }^{[48]}$ 在 1999 年, 就利用微波在不到两分钟内使正己烷-2,5-二酮 与伯胺发生 Knorr-Paal 缩合反应, 生成一系列 $N$-取代吡 咯衍生物. 因而, 我们认为也可通过微波诱导先生成吡 咯衍生物, 然后卤代, 再将噻吩基通过偶联反应引入到 吡咯环中从而得到 2,5-二噻吩基吡咯衍生物.

\section{3 应用}

\section{1 在电致变色器件方面的应用}

2,5-二噻吩基吡咯衍生物作为电致变色活性材料, 具有可溶解性、高电导率、高稳定性以及较高的储存电 荷能力 ${ }^{[49,50]}$, 易于通过化学方法和电化学方法合成, 是 目前最有应用前景的功能材料之一. 由于电致变色材料 具有双稳态的性能, 所以用电致变色材料做成的电致变 色显示器件不仅不需要背光源, 而且显示静态图象后, 只要显示内容不变化, 就不会耗电, 达到节能的目的. 此外, 研制开发出的多种电致变色器件, 其具有的透光 度可以在较大范围内随意调节, 多色连续变化, 以及存 储记忆功能、驱动变色电压低、受环境影响小等特性, 因 此拥有十分广阔的应用前景.

真正开始 2,5-二噻吩基吡咯衍生物的电化学性能研 究是从 2000 年开始, Brillas 等 ${ }^{[51]}$ 研究了 2,5-二噻吩基吡 咯在 Pt 电极上的电化学行为. 此后 Toppare 和 Cihaner 等 ${ }^{[52 ~ 58]}$ 开始大量合成新型 2,5 -二噻吩基吡咯衍生物, 并 详细研究了其光电化学性能.

2007 年, Yigitsoy 等 ${ }^{[59]}$ 以噻吩为原料合成了 1 -甲苯 基-2,5-二(2-噻吩基)- $1 H$-吡咯(DTTP) (40). 利用电沉积 法在 ITO 导电玻璃上制备聚合物薄膜, 并制备了电致变 色器件, 使聚 (3,4-乙撑二氧噻吩) (PEDOT) 作为阴极变 色层, 其基本结构为 ITO/P(DTTP) ||gel electrolyte||PEDOT/ITO, 且采用紫外可见吸收光谱对它的光 学性能进行了研究, 表明此聚合物在 $-0.6 \sim+1.6 \mathrm{~V}$ 区 间内在绿色和蓝色间循环变化, 光对比度在波长为 617 $\mathrm{nm}$ 处能够达到 $30 \%$. 此外, 制作的器件具有良好的开 路记忆功能及稳定的电致变色性能.

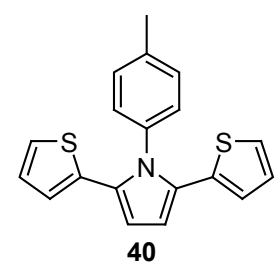

2008 年, Cihaner 等 ${ }^{[60]}$ 成功地合成了 1-(9H-芴-2-基)2,5-二(2-噻吩基)- $1 H$-吡咯(41), 并对制备的电致变色器 件进行了研究. 结果表明此化合物具有较高的着色效
率，高氧化稳定性(在经过 4000 次转换后，仍能保持 $98.6 \%$ 的光学活性)和非常快的响应时间(小于 $0.5 \mathrm{~s}$ ).

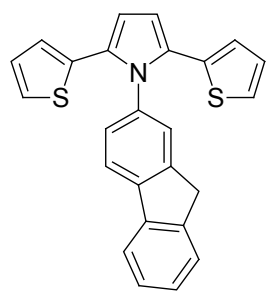

41

2007 年, Tarkuc 等 ${ }^{[61]}$ 以噻吩为原料, 通过 KnorrPaal 缩合反应, 设计合成了 1-芐基-2,5-二(2-噻吩基)$1 H$-吡咯(SNBS) (42), 并制备了电致变色器件, 基本结 构为 $\mathrm{ITO} / \mathrm{P}(\mathrm{SNBS}-\mathrm{co}-\mathrm{EDOT}) \mid$ gel electrolyte||PEDOT/ ITO. 紫外可见吸收光谱分析表明, 在电压为 1.0, 0.3 和 $0.8 \mathrm{~V}$ 时, 可分别产生酒红、绿、蓝三种颜色, 光对比度 在波长为 $485 \mathrm{~nm}$ 处能够达到 $40 \%$. 由于 2,5 -二噻吩基吡 咯衍生物 42 具有响应时间快、颜色变化种类多等优异 性能, 使其在电致变色材料中具有潜在的应用价值.

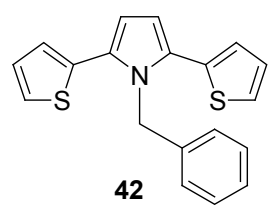

2008 年, $A k$ 等 ${ }^{[62]}$ 合成 1,6-二-(2,5-二噻吩- $1 H$-吡咯1-基)-已烷(TPH)(43). 利用它的高导电性, 良好的氧化 还原可逆性, 且能使颜色迅速转换的性质, 可与具有高 稳定性和低带隙的 EDOT 共聚. 得到的共聚物使其电致 变色性能有了明显的提高, 如光学对比度和响应时间, 以及能产生五种不同的颜色: 紫色、红色、浅灰色、绿 色和蓝色[原本 $\mathrm{P}(\mathrm{TPH})$ 只能产生三种颜色：蓝色、绿色 和黄色]. 这些性质使得该化合物在制作显示器方面显 示出良好的优越性.

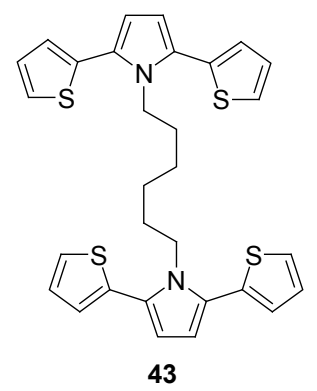

2010 年, 本课题组 ${ }^{[63]}$ 将 1,4-二(2-噻吩基)-1,4-丁二 酮与 1-/2-氨基-葱醌利用 Knorr-Paal 缩合反应，生成 2,5二噻吩基吡咯衍生物 44, 45. 并通过电化学方法合成了 
相应的聚合物薄膜 P44, P45. 紫外可见吸收光谱分析表 明，通过对聚合物薄膜 P44 和 P45 施加不同电压，从 $-1.2 \mathrm{~V}$ 到 $1.2 \mathrm{~V}$, 它们的颜色主要从黄色, 灰色到蓝色 逐渐变化; 在 $A=780 \mathrm{~nm}$ 处测量聚合物薄膜 P44 和 P45 透过率随时间的变化, 表明 P44 和 P45 都具有较快的响 应速率，有望制作响应时间更短的电致变色器件.
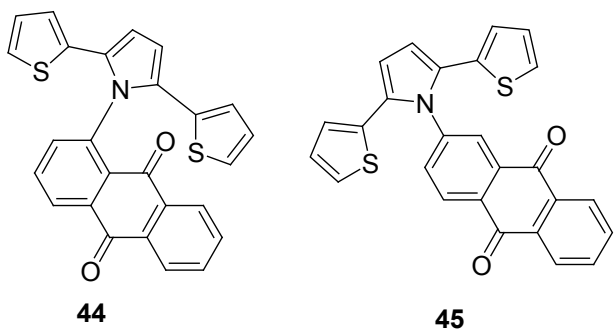

2011 年, 本课题组 ${ }^{[64,65]}$ 以噻吩为起始原料, 设计合 成了含有芳胺基团的 2,5-二噻吩基吡咯衍生物和双 2,5二噻吩基吡咯衍生物等两个系列的 2,5-二噻吩基吡咯衍 生物 46 51. 利用电沉积法在 ITO 导电玻璃上制备聚 合物薄膜 $\mathbf{P 4 6} \sim \mathbf{P 5 1}$, 并用紫外可见分光光度计测量其 光学性能. 通过对聚合物薄膜 P46 P51 施加不同电压, 从-1.2 $\mathrm{V}$ 到 $1.2 \mathrm{~V}$, 它们呈现出多种颜色, 颜色主要从 黄色到灰色, 再到蓝色变化. P46 P51 都具有较低的能 量带陌，是优良的导电聚合物材料.

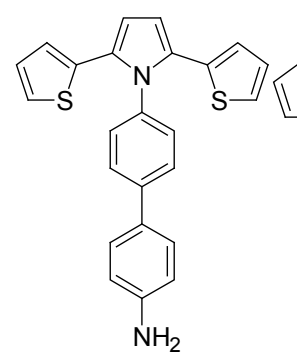

46

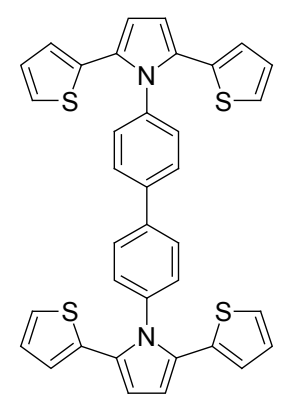

49<smiles>Nc1ccc(Oc2ccc(-n3c(-c4cccs4)ccc3-c3cccs3)cc2)cc1</smiles>

47

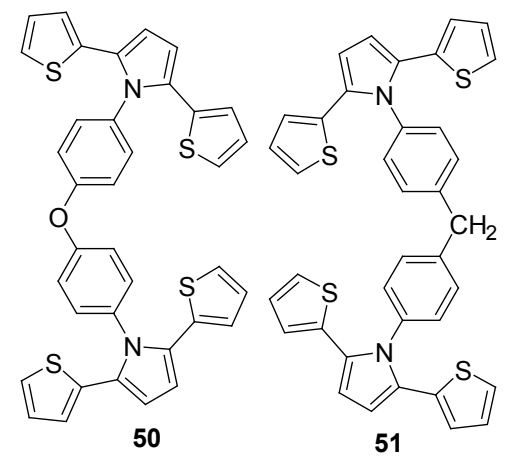

综上所述, 2,5-二噻吩基吡咯衍生物在电致变色材 料领域有着潜在的广泛应用价值: 如有望根据其光谱特 性、良好的稳定性、快速的响应时间等特点制作灵巧窗. 这种智能窗能够对热辐射特别是阳光辐射光谱的某段
谱区产生反射或吸收，可以有效地控制通过窗户的光谱 和能量流，实现对室内光线和温度的调节. 其次, 利用 其良好的开路记忆功能用它们来作为电色材料, 可用来 存储和读出信息. 此外, 可在电致变色器件中设置一个 反射层，通过电致变色的光选择性吸收的特性，调节反 射光线，可以做成无眩反光镜.

\section{2 在光电子器件方面的应用}

由于 2,5-二噻吩基吡咯衍生物是一种有机半导体材 料, 它们的带隙在 $1.5 \sim 3 \mathrm{eV}$ 之间, 符合制造光电子器 件的要求 ${ }^{[66]}$, 并且将其制得的光电子器件响应时间有 望更短. 2001 年, Dhanabalan 等 ${ }^{[67]}$ 报道了新型平面给予 体-受体 $\mathrm{C}_{60}$-2,5-二噻吩基吡咯衍生物的合成. 以 1,4-二 (2-噻吩基)-1,4-丁二酮为原料, 与伯胺通过缩合反应生 成 $N$-十二烷基-2,5-二(2-噻吩基)吡咯, 然后通过 Vilsmeier-Haack 反应对两个噻吩基上的 $\alpha$ 位进行甲酰 化, 得到 $N$-十二烷基-2,5-二(2-噻吩基)-3,4-二醛基吡咯, 再与 $\mathrm{C}_{60}$ 进行 Prato 反应后可得到目标产物 $\mathbf{5 2}$. 以 $\pi$-共 轭的 2,5-二噻吩基吡咯衍生物作为给予体, 富勒烯 $\mathrm{C}_{60}$ 作为受体合成的 $\mathrm{C}_{60}$-2,5-二噻吩基吡咯衍生物 52, 可以 用来制备光电子器件. 研究表明, 富勒烯衍生物的共轭 体系越大，结构越不对称，越有利于非线性光学响 应 $^{[68]}$.

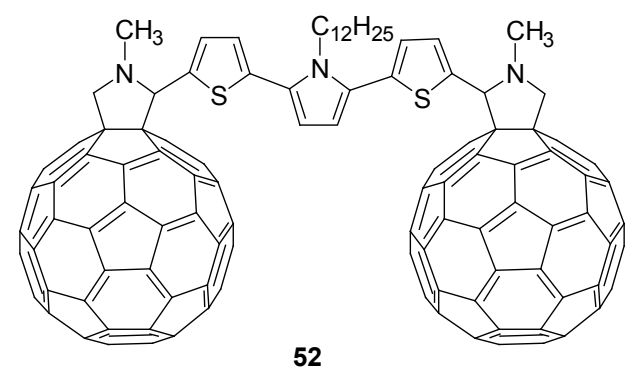

2006 年, Edder 等 ${ }^{[69]}$ 成功制备了 2,5-二噻吩基吡咯 衍生物 53. 由于该化合物含有热裂解基团，烷基链的热 运动会将其不溶性传递给有机材料，进而导致聚合物薄 膜的大量沉积，而隔开的可溶性链的减少也会使这些聚 合物膜的空穴迁移率和吸收系数增加，最终提高有机太 阳能器件的能量转换效率, 所以在光电子器件上有潜在 的应用价值.

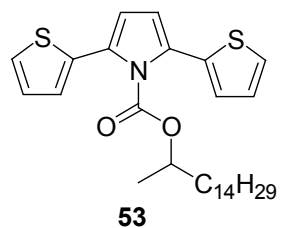

2008 年, Cihaner 等 ${ }^{[49]}$ 设计合成了 1-(1-/2-菒基)-2,5二(2-噻吩基)- $1 H$-吡咯 54, 55. 当施加不同电压于 $\mathbf{5 4 , 5 5}$ 
的聚合物时能显示不同颜色, 比如, 紫外可见吸收光谱 分析表明, 其中 SNS-1-菜可显示黄色(还原态)、绿色(过 渡态)、紫色(氧化态)三种颜色. 另外, 它们的聚合物都 易溶于有机溶剂, 而且都具有苂光性. 苂光分析法表明, 这两种可溶性的聚合物可发出微黄色、绿色的荧光, 有 望应用于有机激光器或者发光二极管.

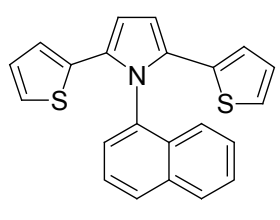

54

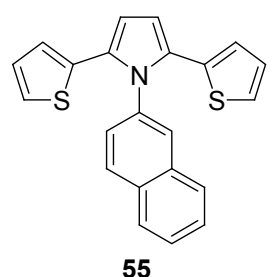

55
2010 年, Yavuz 等 ${ }^{[70]}$ 成功合成了含有四个 2,5 -二噻 吩基吡咯基团的酞化青化合物 56, 且通过电化学方法 得到的聚合物薄膜呈现出良好的可逆氧化还原性. 这两 种聚合物薄膜在中性状态时都呈现为绿色; 在氧化状态 时, $\mathrm{P}\left(\mathrm{H}_{2} \mathrm{Pc}-\mathrm{SNS}\right)$ 呈现为松石绿色, $\mathrm{P}(\mathrm{ZnPc}-\mathrm{SNS})$ 呈现为 黑色. 此外, 苂光测试结果表明, $\mathrm{P}(\mathrm{ZnPc}-\mathrm{SNS})$ 是一个很 好的蓝色和橙色的发光体, 可有望运用于有机激光器或 LED.

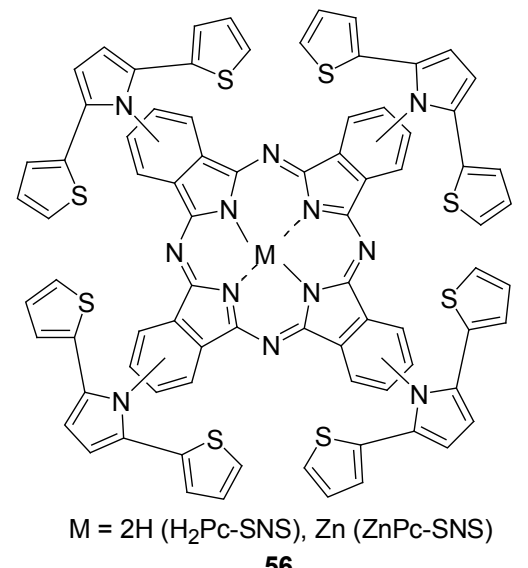

56

\section{3 在传感器方面的应用}

2,5-二噻吩基吡咯衍生物对电信号的变化非常敏 感, 可以用作传感器. 2009 年, Tuncagil 等 ${ }^{[71]}$ 成功地合成 了 4-(2,5-二(2-噻吩基)- $1 H$-吡咯-1-基)苯胺 57. 将该 2,5二噻吩基吡咯衍生物通过电化学方法固定葡萄糖氧化 酶, 当葡萄糖氧化酶使葡萄糖氧化分解时, 产生的过氧 化氢可掺杂 2,5-二噻吩基吡咯衍生物, 从而改变 2,5-二 噻吩基吡咯衍生物膜的电导率. 通过检测 2,5-二噻吩基 吡咯衍生物电导率的变化, 就可以测定实际样品中乙醇 或者葡萄糖的浓度, 生物需氧量(BOD), 有毒物质和吸 收的糖, 以及选择性检测其中一种被测物. 因此, 它是 非常有应用价值的生物传感器.

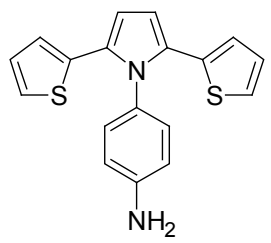

57

Tuncagil 等 ${ }^{[72 ~ 74]}$ 还合成出了 4-[2,5-二(2-噻吩基)$1 H$-吡咯-1-基]硝基苯(58). 将酪氨酸酶包含在硝基中加 以固化, 再用透析膜覆盖后固定在石墨电极的上进行聚 合，便制成了酶传感器. 此方法制备简单、耗时较短. 虽 然酶是非常昂贵的生物催化剂，但是固定化过程是有效 的，固定化酶可以从溶液中恢复，并且重复使用. 如制 成的固定化熫糖酶传感器 ${ }^{[75]}$, 可用于食品行业的糖果 生产中，它可以防止有色副产品的生产过程中的水文裂 解, 而且也使其降低了结晶度 ${ }^{[76]}$. 此外, 2009 年, Cihaner 等 ${ }^{[77]}$ 设计合成了含有双吡咯基团的 2,5-二噻吩基 吡咯衍生物 59. 据 Krondak 等 ${ }^{[78]}$ 报道, 双吡咯聚合物能 够表现出对酸性及碱性气体较高的敏感性, 所以 2,5-二 噻吩基吡咯衍生物 59 有望在人工舌头及人工鼻子作为 信号传导的气敏传感器.
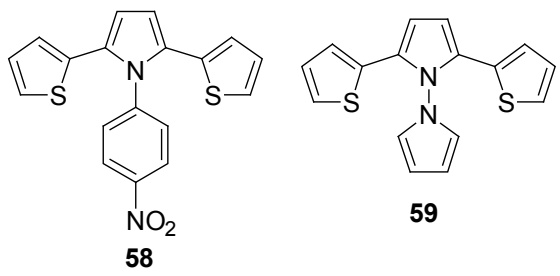

\section{4 其他方面的应用}

根据 2,5-二噻吩基吡咯衍生物的光电特性、可大量 生产、器件制造简单等优点, 有望作为太阳能电池的材 料; 也可根据它们的可加工性，用作防腐蚀或者防静电 涂料; 另外, 由于它们成本低, 不消耗资源, 方便大面 积应用，因此它们也是一种非常理想的电磁屏蔽材料替 代品，可以应用在计算机房、电视机、电脑和心脏起搏 器上. 此外, 最新研究发现, DNA 具有导电性 ${ }^{[79]}$, 因此, 与生命科学相结合, 2,5-二噻吩基吡咯衍生物有望用来 制造人造神经和人造肌肉，以促进 DNA 生长和修饰 DNA. 这将是导电聚合物研究在应用上最重的一个新 趋势.

\section{4 结束语}

随着对 2,5-二噻吩基吡咯类化合物研究的不断深 入，其在有机合成、材料学、生物学、医学等领域将拥 有十分广泛的应用前景. 全面了解 2,5-二噻吩基吡咯衍 
生物有效的合成方法有助于研究者针对不同的目标化 合物设计和选择最为高效简洁的合成策略，从而推动先 导化合物的发现与结构优化. 此外, 近年来研究发现, 大多数的 2,5-二噻吩基吡咯衍生物都能显示出良好的光 电化学性能: 它们的聚合物兼有聚噻吩和聚吡咯的共同 优点, 其较低的氧化电位、较低的能隙及良好的导电性 能、光化学性能、电致变色性能、稳定性能、成膜性能 等优良性能使其在电致变色器件、光电子器件、传感器 等方面有广泛的应用前景. 在现实应用上, 2,5-二噻吩 基吡咯衍生物目前还处在突破的前夜, 大多数还处于实 验室研究阶段, 真正的实用化还未取得质的进步, 需要 进一步研究. 今后研究发展的关键在于其性能、价格以 及市场需要与无机材料竞争; 稳定性需要加强, 脱掺杂 问题亟待解决; 加工性能和力学性能有待提高. 如果这 些问题都能在规模化应用之前得到圆满解决, 我们相 信, 随着对 2,5-二噻吩基吡咯衍生物的深入研究和开发, 预计以导电聚合物和功能有机分子材料为主体的有机 电子材料和器件, 将在无机半导体材料之后带来另一场 新的技术革命.

\section{References}

[1] Chiang, C. K.; Fincher, C. R. Jr.; Park, Y. W.; Heeger, A. J.; Shirakawa, H.; Louis, E. J.; Gau, S. C.; MacDiarmid, A. G. Phys. Rev. Lett. 1977, 39, 1098.

[2] Ito, T.; Shirakawa, H.; Ikeda, S. J. Polym. Sci. Part A: Polym. Chem. 1974, 12, 11.

[3] Shirakawa, H.; Lowis, E. J.; MacDiarmid, A. G.; Chiang, C. K.; Heeger, A. J. J. Chem. Soc., Chem. Commun. 1977, 16, 578.

[4] Chiang, C. K.; Druy, M. A.; Gau, S. C.; Heeger, A. J.; Louis, E. J.; Macdiarmid, A. G.; Rark, Y. W.; Shirakawa, H. J. Am. Chem. Soc. 1978, 100, 1013.

[5] Feng, X. M.; Huang, X. W.; Tan, Z.; Zhao, B.; Tan, S. T. Acta Chim. Sinica 2011, 69, 653 (in Chinese). (冯小明, 黄先威, 谭卓, 赵斌, 谭松庭, 化学学报, 2011, 69, 653.)

[6] Deng, M.; Wang, M.; Chen, H. Z. Acta Chim. Sinica 2011, 69, 477 (in Chinese) (邓萌, 汪茫, 陈红征, 化学学报, 2011, 69, 477.)

[7] Hill, R. M. Curr. Opin. Colloid Interface Sci. 2002, 7, 255.

[8] Jin, S.; Cong, S.; Xue, G.; Xiong, H.; Mansdorf, B.; Cheng, S. Z. D. Adv. Mater. 2002, 14, 1492.

[9] Ferraris, J. P.; Skiles, G. D. Polymer 1987, 28, 179.

[10] Koyuncu, S.; Zafer, C.; Sefer, E.; Koyuncu, F. B.; Demic, S.; Kay, I.; Ozdemir, E.; Icli, S. Synth. Met. 2009, 159, 2013.

[11] Hwang, J. Y.; Son, J. I.; Shimn, Y. B. Sol. Energy Mater. Sol. Cells 2010, 94, 1286.

[12] Kubicoval, L.; Waisser, K.; Kunes, J.; Kralova, K. Odlerova, Z.; Slosarek, M.; Janota, J.; Svoboda, Z. Molecules 2000, 5, 714.

[13] Koyuncu, F. B.; Sefer, E.; Koyuncu, S.; Ozdemir, E. Polymer 2011, 52, 5772 .

[14] Atalar, T; Cihaner, A.; Alg1, F. Turk. J. Chem. 2009, 33, 313.

[15] Galindo, M. A.; Hannant, J.; Harrington, R. W.; Clegg, W.; Horrocks, B. R.; Pike, A. R.; Houlton, A. Org. Biomol. Chem. 2011, 9, 1555 .
[16] Hua, W.-T. Chemistry 1984, 10, 31 (in Chinese). (花文廷, 化学通报, 1984, 10,31.)

[17] Broadbent, H. S.; Burnham, W. S.; Qlsen, R. K.; Sheeley, R. M. J. Heterocycl. Chem. 1968, 5, 757.

[18] Zeng, G. -Z. Chin. Sci. Bull. 1978, 616, 2310 (in Chinese). (曾广值, 科学通报, 1978, 616, 2310.)

[19] Just, P. E.; Chane-Ching, K. I.; Lacaze, P. C. Tetrahedron 2002, 58, 3467.

[20] Yildiz, E.; Camurlu, P.; Tanyeli, C.; Akhmedov, I.; Toppare, L. J. Electroanal. Chem. 2008, 612, 247.

[21] Varis, S.; Ak, M.; Tanyeli, C.; Akhmedov, I. M.; Toppare, L. Eur. Polym. J. 2006, 42, 2352

[22] Arslan, A.; Türkarslan, Ö.; Tanyeli, C.; Akhmedov, İ. M.; Toppare, L. Mater. Chem. Phys. 2007, 104, 410.

[23] Nakazaki, J.; Chung, I.; Matsushita, M. M.; Sugawara, T.; Watanabe, R.; Izuoka, A.; Kawada, Y. J. Mater. Chem. 2003, 13, 1011.

[24] Niziurski-Mann, R. E.; Scordilis-Kelley, C.; Liu, T. L.; Cava, M. P.; Carlin, R. T. J. Am. Chem. Soc. 1993, 115, 887.

[25] Sorensen, A. R.; Overgaard, L.; Johannsen, I. Synth. Met. 1993, 55, 1626.

[26] Ogura, K.; Yanai, H.; Miokawa, M.; Akazome, M. Tetrahedron Lett. 1999, 40, 8887.

[27] Niziurski-Mann, R. E.; Cava, M. P. Adv. Mater. 1993, 5, 718.

[28] Fujii, M.; Nishinaga, T.; Iyoda, M. Tetrahedron Lett. 2009, 50, 555.

[29] Minh, T. N.; Asit, K. C.; Shogo, S.; Keiji, M. J. Org. Chem. 1999, 64,65 .

[30] St-Cyr, D. J.; Morin, M. S. T.; Belanger-Gariepy, F.; Arndtsen, B. A.; Krenske, E. H.; Houk, K. N. J. Org. Chem. 2010, 75, 4261.

[31] St-Cyr, D. J.; Arndtsen, B. A. J. Am. Chem. Soc. 2007, 129, 12366.

[32] Naka, H.; Koseki, D.; Kondoa, Y. Adv. Synth. Catal. 2008, 350, 1901.

[33] Zheng, Q. W.; Hua, R. M. Tetrahedron Lett. 2010, 51, 4512.

[34] Dou, G.; Shi, C.; Shi, D. J. Comb. Chem. 2008, 10, 810.

[35] Kende, A. S.; Roth, B.; Sanfilippo, P. J.; Blacklock, T. J. J. Am. Chem. Soc. 1982, 104, 5808.

[36] Ono, N.; Yamamoto, T.; Shimada, N.; Kuroki, K.; Wada, M.; Utsunomiya, R.; Yano, T.; Uno, H.; Murashima, T. Heterocycles 2003, 61, 433.

[37] Dang, T. T.; Ahmad, R.; Dang, T. T.; Reinke, H.; Langer, P. Tetrahedron Lett. 2008, 49, 1698

[38] Byrne, P. D.; Muller, P.; Swager, T. M. Langmuir 2006, 22, 10596.

[39] Kang, J.-G.; Oh, S.; Cho, D.-H.; Nah, M.-K.; Park, C.; Bae, Y. J.; Han, W. T.; Park, Y. J.; Lee, S. W.; Kim, I. T. Bull. Korean Chem. Soc. 2009, 30, 1157

[40] Lehuede, J.; Mettey, Y.; Vierfond, J.-M. Synth. Commun. 1996, 26, 793.

[41] Merrifield, R. B. J. Am. Chem. Soc. 1963, 85, 2149

[42] Wang, D. X. Solid-Phase Organic Synthesis: Principle and Application Guide, Chemical Industry Press, Beijing, 2004, p. 2 (in Chinese).

(王德心，固相有机合成一原理及应用指南，化学工业出版社，北 京, 2004, p. 2.)

[43] Martin, B.; Sekljic, H.; Chassaing, C. Org. Lett. 2003, 5, 1851.

[44] Yang, G.-C.; Chen, Z.-X.; Hu, C.-L. Chin. J. Org. Chem. 2002, 22, 289 (in Chinese) (杨桂春, 陈祖兴, 胡春玲, 有机化学, 2002, 22, 289.)

[45] Kobayashi, N.; Kaku, Y.; Higurashi, K.; Yamauchi, T.; Ishibashib, A.; Okamoto, Y. Bioorg. Med. Chem. Lett. 2002, 12, 1747.

[46] Gedye, R.; Smith, F.; Westway, K.; Ali, H.; Baldisera, L.; Laberge, L.; Rousell, J. Tetrahedron Lett. 1986, 27, 279.

[47] Gedye, R.; Smith, F.; Westway, K. Can. J. Chem. 1987, 66, 17. 
[48] Danks, T. N. Tetrahedron Lett. 1999, 40, 3957.

[49] Cihaner, A.; Alg1, F. Electrochim. Acta 2008, 54, 665.

[50] Tarkuc, S.; Sahmetlioglu, E.; Tanyeli, C.; Akhmedov, I. M.; Toppare, L. Opt. Mater. 2008, 30, 1489.

[51] Brillas, E.; Carrasco, J.; Oliver, R.; Estrany, F.; Vilar, J.; Morlans, J. M. Electrochim. Acta 2000, 45, 4049.

[52] Varis, S.; Ak, M.; Akhmedov, I. M.; Tanyeli, C.; Toppare, L. Electrochim. Acta 2007, 52, 6561.

[53] Varis, S.; Ak, M.; Akhmedov, I. M.; Tanyeli, C.; Toppare, L. J. Electroanal. Chem. 2007, 603, 8.

[54] Camurlu, P.; Tarkuç, S.; Şahmetlioğlu, E.; Akhmedov, İ. M.; Tanyeli, C.; Toppare, L. Sol. Energy Mater. Sol. Cells 2008, 92, 154.

[55] Kiralp, S.; Camurlu, P.; Gunbas, G.; Tanyeli, C.; Akhmedov, I.; Toppare, L. J. Appl. Polym. Sci. 2009, 112, 1082.

[56] Cihaner, A.; Alg1, F. J. Electroanal. Chem. 2008, 614, 101.

[57] Cihaner, A.; Alg1, F. Electrochim. Acta 2009, 54, 1702.

[58] Cihaner, A.; Alg1, F. Electrochim. Acta 2008, 54, 786.

[59] Yigitsoy, B.; Varis, S.; Tanyeli, C.; Akhmedov, I. M.; Toppare, L. Thin Solid Films 2007, 515, 3898.

[60] Cihaner, A.; Alg1, F. Electrochim. Acta 2008, 53, 2574.

[61] Tarkuc, S.; Sahmetlioglu, E.; Tanyeli, C.; Akhmedov, I. M.; Toppare, L. Sens. Actuators, B 2007, 121, 622.

[62] Ak, M.; Sahmetlioglu, E.; Toppare, L. Electroanal. Chem. 2008, 612,55 .

[63] Wang, G.; Fu, X. K.; Huang, J.; Wu, L.; Du, Q. L. Electrochim. Acta 2010, 55, 6933.

[64] Wang, G.; Fu, X. K.; Hang, J.; Wu, L.; Deng, J. J. Electroanal. Chem. 2011, 661, 351.

[65] Wang, G.; Fu, X. K.; Hang, J.; Wu, C. L.; Wu, L.; Deng, J.; Du, Q.
L.; Zou, X. C. Electrochim. Acta 2011, 56, 6352.

[66] Song, X.-Q. Recent Dev. Sci. Technol. Abroad 2001, 10, 26 (in Chinese).

(宋心琦, 国外科技动态, 2001, 10, 26).

[67] Dhanabalan, A.; Knol, J.; Hummelen, J. C.; Janssen, R. A. J. Synth. Met. 2001, 119, 519.

[68] Liu, S.-Z.; Tang, G.-S. Prog. Chem. 2004, 16, 561 (in Chinese). (刘书芝, 唐光诗, 化学进展, 2004, 16, 561).

[69] Edder, C.; Armstrong, P. B.; Prado, K. B.; Frechet, J. M. J. Chem. Commun. 2006, 18, 1965.

[70] Yavuz, A.; Bezgin, B.; Aras, L.; Önal, A. M. J. Electroanal. Chem. 2010, 639, 116.

[71] Tuncagil, S.; Odaci, D.; Yildiza, E.; Timurb, S.; Topparea, L. Sens. Actuators, B 2009, 137, 42.

[72] Tuncagil, S.; Kiralp, S.; Toppare, L. React. Funct. Polym. 2008, 68, 710.

[73] Tuncagil, S.; Odaci, D.; Varis, S.; Timur, S.; Toppare, L. Bioelectrochemistry 2009, 76, 169.

[74] Tuncagil, S.; Varis, S.; Toppare, L. J. Mol. Catal. B: Enzym. 2010, $64,195$.

[75] Underkofler, L. A.; Barton, R. R.; Rennet, S. S. Appl. Microbiol. 1958, 6, 212.

[76] Selampinar, F.; Akbulut, U.; Ozden, M. Y.; Toppare, L. Biomaterials 1997, 18, 1163.

[77] Cihaner, A.; Mert, O.; Demir, A. S. Electrochim. Acta 2009, 54, 1333.

[78] Krondak, M.; Broncová, G.; Anikin, S.; Merz, A.; Mirsky, V. M. J. Solid State Electrochem. 2006, 10, 185.

[79] Murphy, C. J.; Arkin, M. R.; Jenkins, Y.; Ghatlia, N. D.; Bossman, S. H.; Turro, N. J.; Barton, J. K. Science 1993, 262, 1025.

(Cheng, F.) 